\title{
Hemipelvectomia total em um gato com osteossarcoma osteoblástico
}

\author{
Total Hemipelvectomy in a Cat with Osteoblastic Osteosarcoma
}

\author{
Maria Thereza Bonfim Ens' ${ }^{1}$ Matias Bassinello Stocco ${ }^{2}$, Lianna Ghisi Gomes ${ }^{3}$, Luciana Maria Curtio Soares ${ }^{4}$, \\ Nathalie Moro Bassil Dower ${ }^{3}$, Camila Calvi Menegassi ${ }^{3}$, Luciana Dambrósio Guimarães ${ }^{5}$, \\ Edson Moleta Colodel ${ }^{5} \&$ Roberto Lopes de Souza ${ }^{5}$
}

\begin{abstract}
Background: Osteosarcoma (OSA) is a malignant neoplasm of mesenchymal tissue that exhibits various histological features and a differentiated biological clinical behavior.The combination of clinical, radiological, and histopathological features is important for diagnosis. Surgical treatment of neoplasms with pelvic involvement is a challenge.Primary bone tumors are rare in cats and are mostly reported in older animals. The objective of this paper was to report the case of a feline subjected to total hemipelvectomy as primary treatment of pelvic OSA; the surgical technique and histological analysis of the tumor are described.No signs of recurrence were seen during seven months of follow-up.

Case: A 4-year-old female mixed breed cat weighing $3 \mathrm{~kg}$ was presented with a one year history of increased volume on the hip joint region. Palpation of the increased volume on the hip showed a firm, adhered, and painful mass. Radiographic examination of the pelvis in the dorso ventral projection exhibited a severe osteoblastic bone reaction on the proximal femur, as well as marked periosteal reaction of the hip joint with involvement of the acetabulum. A ventral approach total hemipelvectomy technique was adopted for excision of the tumor. The animal was placed on a lateral position and a circumferential skin incision was performed on the ipsilateral limb of the affected hemipelvis, on the medial portion of the femur, and was extended cranially up to the thoracolumbar region. The pubic symphysis was exposed with a periosteal elevator and a pubic symphysiotomy was performed on the midline using a Liston bone cutter; next, the sacroiliac junction was resected. No drain was used after surgery, only compressive bandages. The patient was hospitalized for post-operative monitoring which included opioids for pain management and a non-steroidal anti-inflammatory drug and a cephalosporin class antibiotic. The wound was cleaned with saline, rifamycin spray (Rifocina Spray ${ }^{\circledR}$ ) was applied, and dressing was changed every $12 \mathrm{~h}$. The excised bone was submitted for pathological analysis. Gross examination revealed a 4.2 $\mathrm{cm}$ hardened increase in volume on the area of the femoral head and acetabulum, with irregular surface; cut surface was hard and white with black and red spots. Histology revealed osteoblastic OSA with intense and solid fusiform and stellate cells distributed in a solid pattern with no capsular border, with marked infiltration of the bone tissue, marked osteolysis, moderate proliferation of osteoclasts, and moderate anisocytosis and anisokaryosis.

Discussion: Bone tumors in cats are rare and develop spontaneously.They exhibit aggressive behavior and promote local bone destruction.Firm consistency and local swelling usually occur in more advanced cases when there is a delay between the onset of clinical signs and the veterinary consultation. Radiological findings such as bone destruction and bone formation are commonly seen in this type of tumor. Hemipelvectomy is considered an aggressive surgical technique, but necessary in many cases, especially when tumors are located in the pelvis and nearby tissues. In the case presented here, this surgery was the most viable and advised therapeutic option because of tumor location. No incidents (i.e. hemorrhage) occurred during the perioperative period. After surgery, the patient continued to perform all custom functions. The hind limb, which was affected in this case, is commonly reported to be affected in the literature; however, it is unusual to have involvement of both bones (pelvis and femur) since vascularization of these bones are independent.Diagnosis was confirmed by histopathology in which a characteristic malignant mesenchymal tumor of primitive bone cells was present, with production of osteoid of variable shapes and quantity with the presence of osteoclastic giant cells.
\end{abstract}

Keywords: neoplasia, bone, cat, pelvis, histopathology.

Descritores: neoplasia, osso, gato, pelve, histopatologia.

${ }^{1}$ M.V., Universidade Federal de Mato Grosso (UFMT), Cuiabá, MT, Brazil. ${ }^{2}$ Doutorado \& ${ }^{3}$ Mestrado, Programa de Pós-graduação em Ciências Veterinárias, UFMT, Cuiabá. ${ }^{4}$ Residente, Programa Uniprofissional em Medicina Veterinária, UFMT, Cuiabá. ${ }^{5}$ Departamento de Clínica Médica Veterinária, Faculdade de Veterinária (FAVET), UFMT, Cuiabá. CORRESPONDENCE: R.L. Souza [lopesdesouza.roberto@ gmail.com - Tel.: +55 (65) 3615-8662]. Hospital Veterinário, Universidade Federal de Mato Grosso-UFMT. Avenida Fernando Côrrea da Costa n²367. CEP 78060-900 Cuiabá, MT, Brazil. 


\section{INTRODUÇÃO}

O osteossarcoma (OSA) faz parte de um grupo heterogênio de neoplasias malignas, que afetam a formação de osso ou tecido mesenquimal [11]. Tumores ósseos primários são raros em felinos, acomentendo principalmente animais idosos [8]. A classificação histológica é dada como: osteoblástico, condroblástico, fibroblástico, rico em células gigantes ou telangectásico, dependendo do elemento celular dominante [11]. A combinação de sinais clínicos, localização tumoral, radiografia e histopatologia são importantes no diagnóstico [2]. Tratamento de neoplasia associada à pelve e membro pélvico é um desafio cirúrgico [9]. A hemipelvectomia é agressiva, mas pode ser necessária para se conseguir margens de segurança livres de tumor[1].

São infrequentes os casos de OSA em felinos e como tratamento primário a realização de hemipelvectomia total, objetivou-se relatar a técnica cirúrgica e a confirmação do diagnóstico histopatológico.

\section{CASO}

Felino, fêmea, 4 anos, sem raça definida, pesando $3 \mathrm{~kg}$ e apresentando aumento de volume na região da articulação coxofemural esquerda com aproximadamente 1 ano. $\mathrm{O}$ aumento de volume apresentava-se aderido, firme e com dor a palpação. Ao exame radiográfico da pelve (Figura 1A) observou-se severa reação óssea do tipo blástica em terço proximal de fêmur esquerdo, acentuada reação periosteal na articulação coxofemoral esquerda, envolvendo acetábulo. As imagens radiográficas do tórax e ultrassonográficas abdominal não sugeriram evidências de metástase. Ao hemograma e avaliação de função hepática e renal, não foram demonstrados parâmetros alterados.

Para a exérese da neoplasia, realizou a hemipelvectomia total a partir do acesso ventral [1]. O animal foi posto em decúbito lateral onde uma incisão na pele foi feita circunferencial no membro ipsilateral da hemipelve afetada, ao nível medial do fêmur e estendido cranialmente até a região toracolombar. Após divulsionar os músculos da região, a artéria e veia femoral foram isoladas e ligados separadamente com fio inabsorvível monofilamentar, nylon 2-0, por ligadura circunferencial. A sínfise pélvica foi exposta com elevador periosteal e a sinfisiotomia pélvica com um cisalha de Liston na linha media e após, fez-se a desarticulação sacro-ilíaca. A miorrafia foi realizada com fio nylon 2-0, pontos simples separado, reduzindo o espaço morto com categute 2-0, seguido de dermorrafia com nylon 2-0. Optou-se por não colocar dreno, somente bandagem compressiva. $\mathrm{O}$ material foi encaminhado para análise (Figura 1B) onde notou-se aumento de volume na região da cabeça do fêmur e acetábulo de 4,2 cm de diâmetro, consistência endurecida, superfície irregular, ao corte era enrijecida e com superfície brancacenta, manchas enegrecidas e avermelhadas onde se distribuíram de forma coalescente. Caracterizou-se osteossarcoma osteoblástico (Figura 1C) devido à proliferação intensa e sólida de células fusiformes e estreladas que se distribuíram em padrão sólido sem limite capsular e forte infiltração no tecido ósseo além de intensa osteólise com moderada proliferação de osteoclastos e moderada anisocitose e anisocariose.

Após a cirurgia o paciente foi internado para acompanhamento e controle analgésico com opioide, antiinflamatório e utilizou também uma cefalosporina como antibiótico de escolha. As trocas de curativo foram realizadas utilizando solução fisiológica e borrifando Rifocina Spray® ${ }^{1}$ por 15 dias. Após 7 meses da cirurgia, (Figura 1D) o paciente foi submetido a reavaliação radiográfica e ultrassonográfica a cada dois meses, onde não foi constatado qualquer sinal de metástase pulmonar e em órgãos adjacentes ou recidiva local do tumor.

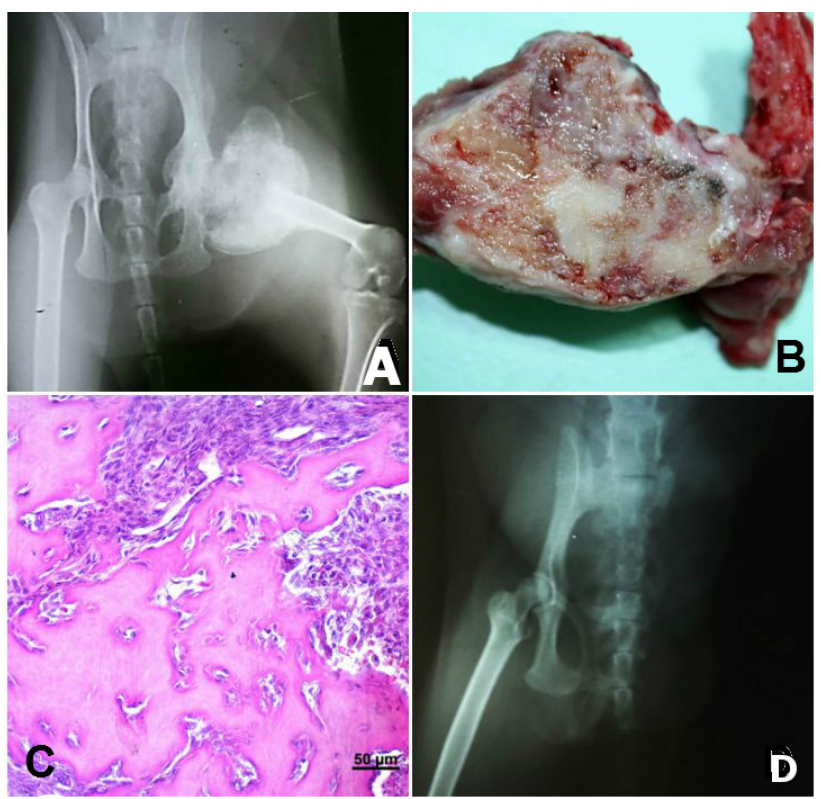

Figura 1. Felino com Osteossarcoma. A- Imagem radiográfica ao momento da consulta com severa reação óssea do tipo blástica em terço proximal de fêmur e acentuada reação periosteal de articulação coxofemoral esquerda, envolvendo acetábulo. BAo corte: endurecida, com superfície brancacenta, manchas enegrecidas e avermelhadas que se distribuem de forma coalescente. C- OSA osteoblástico com proliferação intensa e sólida de células fusiformes e estreladas que se distribuíram em padrão sólido sem limite capsular [Barra $=50 \mu \mathrm{m}]$. D- Sete meses após pós operatório. 


\section{DISCUSSÃO}

Tumores ósseos em felinos são agressivos e promovem destruição óssea local [6] como observado no caso apresentado. Os sinais primários relatados como claudicação, ao início da consulta [7] demonstram serem característicos devido à dor [3]. O aspecto de consistência firme [7] aumento de volume no local, observado pelo caso, acontece em casos mais avançados [3] devido ao tempo de espera entre o inicio do problema e o surgimento dos sinais clínicos. Evidências radiológicas como destruição e formação óssea são características comumente evidenciadas desse tumor como lise e perda da continuidade óssea [6] onde também foram observadas nesse caso. O membro posterior que foi afetado nesse caso, corrobora com a alta incidência descrita na literatura [8] porém torna-se incomum a ocorrência em dois ossos (pelve e fêmur), devido as vascularizações serem independentes. A confirmação do diagnóstico corrobora com a literatura [4], que confirma sendo um tumor mesenquimal maligno de células ósseas primitivas com produção de osteóide [10] onde a forma e quantidade de matriz osteóide é variável, onde foi encontrado células gigantes multinucleadas do tipo osteoclastos [5], como no presente caso.

A hemipelvectomia é considerada uma técnica cirúrgica agressiva e necessária em vários casos, principalmente em tumores localizados na pelve e tecidos próximos [7,9]. No caso do paciente acompanhado, devido o local, tornou-se a opção terapêutica mais viável e aconselhada. A mesma técnica é relatada [1], mas vai de desencontro quanto ao pós operatório e suas complicações. Não houveram interocorrências durante o trans operatório relatado por outros autores [9], como hemorragias e o paciente continuou todos os hábitos de costumes. Devido a técnica ter sido realizada de forma rápida e segura, não houve perda de sangue suficiente para necessidade de transfusão sanguinea, indo de desencontro com outros autores [7,9]. De acordo com o acompanhamento pós operatório do animal e seu estado de saúde geral atual, ressalta-se que a hemipelvectomia total foi a melhor opção para o paciente diagnosticado com o OSA em fêmur com infiltrado em pelve, não havendo assim, recidivas tumorais no local ou adjacentes, após sete meses de procedimento. Levando em consideração que pouco se sabe quanto o comportamento tumoral em felinos, destaca-se sua importância quanto a avaliação criteriosa de todas as etapas.

\section{MANUFACTURER}

${ }^{1}$ Sanofi-Aventis Farmacêutica Ltda. Suzano, SP, Brazil.

Declaration of interest. The authors report no conflicts of interest. The authors alone are responsible for the content and writing of the paper.

\section{REFERENCES}

1 Barbur L.A., Coleman K.D., Schmiedt C.W. \& Radlinsky M.G. 2015. Description of the Anatomy, Surgical Technique, and Outcome of Hemipelvectomy in 4 Dogs and 5 Cats. Veterinary Surgery. 44(5): 613-626.

2 Barger A., Graca R., Bailey K., Messick J., Lorimier L.P \& Fan T. 2005. Use of alkaline phosphatase staining to differentiate canine osteosarcoma from other vimentin-positive tumors. Veterinary Pathology. 42: 161-165.

3 Camargo O.P. \& Baptista A.M. 2004. Conduta atual nas lesões ósseas metastáticas. Revista Brasileira de Ortopedia. 39(6): 273-281.

4 Dimopoulou M., Kirpensteijn J., Moens H. \& Kik M. 2008. Histologic Prognosticators in Feline Osteosarcoma: A Comparison with Phenotypically Similar Canine Osteosarcoma. Veterinary Surgery. 37: 466-471.

5 Fielder S.E. 2013. The Musculoskeletal System. In: Cowell R.L. \& Valenciano A.C. (Eds). Cowell and Tyler's diagnostic cytology and hematology of the dog and cat. 4th edn. St. Louis: Elsevier, pp.216-221

6 Gardinalli B. \& Martelli A. 2014. Aspectos clínicos e fisiopatológicos de osteossarcoma em cães. Science and Animal Health. 3(1): 13-30.

7 Izquierdo D.F.C., Ferrigno C.R.A., Rizzo M.F.C.I., Dal B.Ó.I., Valente A.F., Dos Santos J.F., Poletto M.F., Della Nina M.I., Cavalcanti R.A.O. \& Ferraz V.C.M. 2014. Technical: Hemipelvectomy in cats with pelvic osteosarcoma. Case report. Veterinaria (Montevideo). 50(194): 49-58.

8 Kleiner J.A. \& Silva E.G. 2003. Tumores ósseos em pequenos animais. Medvep. 1(3): 193-200.

9 Kramer A., Walsh P.J. \& Seguin B. 2008. Hemipelvectomy in dogs and cats: technique overview, variations, and description. Veterinary Surgery. 37(5): 413-419. 
M.T.B. Ens, M.B. Stocco, L.G. Gomes, et al. 2017. Hemipelvectomia total em um gato com osteossarcoma osteoblástico. Acta Scientiae Veterinariae. 45(Suppl 1): 190.

10 Lima J.J.G., Manzi F.R., Silva F.L. \& Baptista M.Z. 2002. Osteossarcoma extra-esquelético na região cervical - revisão da literatura e relato de caso clínico. Radiologia Brasileira. 35(5): 315-319.

11 Martelli A., Teixeira L.B.C. \& Santos Jr. A.R. 2007. Aspectos histopatológicos e histoquímico de osteossarcomas em cães. Estudos de Biologia: Ambiente e Diversidade. 29(67): 179-189. 